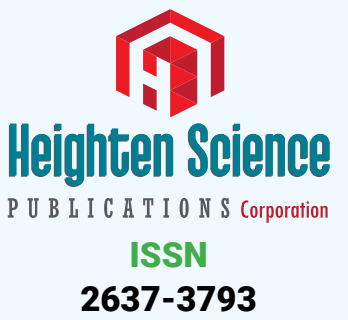

*Address for Correspondence: Paulo Kauffman, Department of Surgery, School of Medicine, University of São Paulo, Brazil, Avenida Nove de Julho, 3229. São Paulo/SP, Brazil, Zip Code: 01407-000, Tel: 5511 3887-8887; Email: kauffman@terra.com.br

Submitted: 16 May 2019

Approved: 03 June 2019

Published: 04 June 2019

Copyright: @ 2019 Kauffman P, et al. This is an open access article distributed under the Creative Commons Attribution License, which permits unrestricted use, distribution, and reproduction in any medium, provided the original work is properly cited

Keywords: Aneurysm; False; Brachial artery; Median nerve; Peripherally inserted central catheter

Check for updates
Case Report

\section{Brachial pseudoaneurysm associated with median nerve injury as a complication of peripherally inserted central catheter: A case report}

\author{
Paulo Kauffman ${ }^{1 *}$, Alfredo S Helito², Mairy JA Poltronieri³, \\ Marcos R Garcia ${ }^{4}$ and Publio CC Viana ${ }^{5}$ \\ 'Department of Surgery, School of Medicine, University of São Paulo, Brazil \\ ${ }^{2}$ General practitioner, Hospital Sírio Libanês, São Paulo, Brazil \\ ${ }^{3}$ Nursing Development and Vascular Access Group Coordinator, Hospital Sírio Libanês, São \\ Paulo, Brazil \\ ${ }^{4}$ Vascular Access Group, Hospital Sírio Libanês, São Paulo, Brazil \\ ${ }^{5}$ Tomography Department Coordinator, Hospital Sírio Libanês, São Paulo, Brazil. Interventional \\ Radiology Service Coordinator, Cancer Institute of São Paulo (ICESP-HC-FMUSP), Brazil
}

\section{Abstract}

Introduction: Peripherally inserted central venous catheters (PICCs) have been increasingly used as an alternative to conventional central venous catheters for long-term administration of chemotherapy, antibiotics, parenteral nutrition, and hydration in patients with difficult venous access. Traumatic complications to arteries and nerves adjacent to veins selected for PICC placement have been rarely described.

Case presentation: We report the case of a PICC placement in the brachial vein of the right upper limb of a 78-year-old woman that resulted in brachial artery pseudoaneurysm and median nerve lesion.

Discussion: The pseudoaneurysm was successfully repaired with thrombin injection, but neurological deficits to the hand resulting from nerve injury persisted even four months after the procedure.

\section{Introduction}

Peripherally inserted central catheters (PICCs) have been used for over 30 years in the United States and more than 15 years in England. PICCs are inserted percutaneously into a peripheral vein with the tip inserted in the superior vena cava. Currently, PICCs are more frequently used as an alternative to central venous catheters (CVCs) in patients who undergo intravenous therapy for more than six days, in those with difficult venous access and in patients undergoing chemotherapy or requiring parenteral nutrition, prolonged antibiotic therapy, hydration, or transfusion of blood and blood products.

The advantages of PICCs include lower cost compared to centrally inserted catheters, safe bedside placement, extended use time, no reported pneumothorax during insertion, less patient discomfort, and low rates of infection and complications.

PICCS are usually inserted by specially trained certified nurses who perform this procedure safely and efficiently. The main adverse events associated with PICCs 
include superficial thrombophlebitis and deep vein thrombosis [1]. Systematic review and meta-analysis studies indicate a $2.7 \%$ risk of venous thromboembolism associated with the use of PICCs [1]. Complications associated with trauma to vessels or nerves adjacent to deep veins punctured for PICC placement are rare, but can result in significant injuries to the patient [2,3], including increasing the risk of infection and venous thrombosis.

At Hospital Sírio Libanês, São Paulo, Brazil, PICCs have been inserted by a group of nine specially trained certified nurses, two of whom work exclusively on PICC line placement, to ensure the procedure is performed safely and efficiently. The catheter is inserted by the modified Seldinger technique using maximum barrier precautions and ultrasound guidance to visualize the vessels. Approximately 3,000 punctures were performed by the group in a two-year period with a success rate of $98 \%$.

This report describes a case of pseudoaneurysm of the brachial artery associated with median nerve injury as a complication of PICC placement. To our knowledge, this is the first nonlegal published case to describe this association of complications after placement of peripherally inserted central catheters.

\section{Case Presentation}

A 78-year-old woman with a history of hypertension, coronary artery disease and permanent pacemaker, chronic renal failure, and who was undergoing dialysis three times a week for two years by arteriovenous fistula in the left upper limb required the insertion of a PICC because of difficulty in obtaining venous access for antibiotic therapy (for 14 days) to treat recurrent episodes of bilateral maxillary sinusitis manifesting as cough, rhinorrhea with purulent sputum, fever, and poor general condition that did not respond to oral treatment.

The PICC was inserted into the brachial vein of the upper right limb in a single puncture by a nurse in the PICC group at Sírio Libanês Hospital, São Paulo, Brazil, after local anesthesia and using ultrasound to guide insertion. There was difficulty in advancing the dilator and pain was reported during the procedure, but catheter insertion was successful. Immediately after insertion, the patient complained of severe pain, mainly in the forearm and hand, had difficulty moving her hand and fingers and a large hematoma formed around the insertion site a few hours after the procedure. No hematoma pulsatility or murmur on auscultation was observed. The right radial and ulnar pulses were normal and the hand was colored and normothermic. The patient had difficulty flexing the forefinger and thumb and was unable to perform prehension movements with the two fingers (Figure $1 \mathrm{AB}$ ). A Doppler ultrasonography of the right hand revealed a pseudoaneurysm of the brachial artery (Figure 2AB). After the pseudoaneurysm diagnosis performed by ultrasonography study, a vascular specialist assessment was request and the catheter was removed. The treatment indicated was thrombin injection into the pseudoaneurysm sac, which was successfully performed (Figure 2CD). The hematoma on the right arm slowly subsided, but the neurological deficit to the right hand remained unchanged after four months of follow-up, despite intensive physical therapy.

\section{Discussion}

The improvement in the quality of PICC materials, the evolution in insertion techniques, the advantages in the use of PICCs, and the presence of nursing staff specialized in PICC placement have resulted in increased use of PICCs over central venous catheters. The advantages of PICCs include bedside placement, easy removal, favorable cost-benefit ratio, and decreased risk of pneumothorax, hemothorax, and air embolism [1].

Anatomically, the vascular bundle of the arm is composed of the brachial artery, 


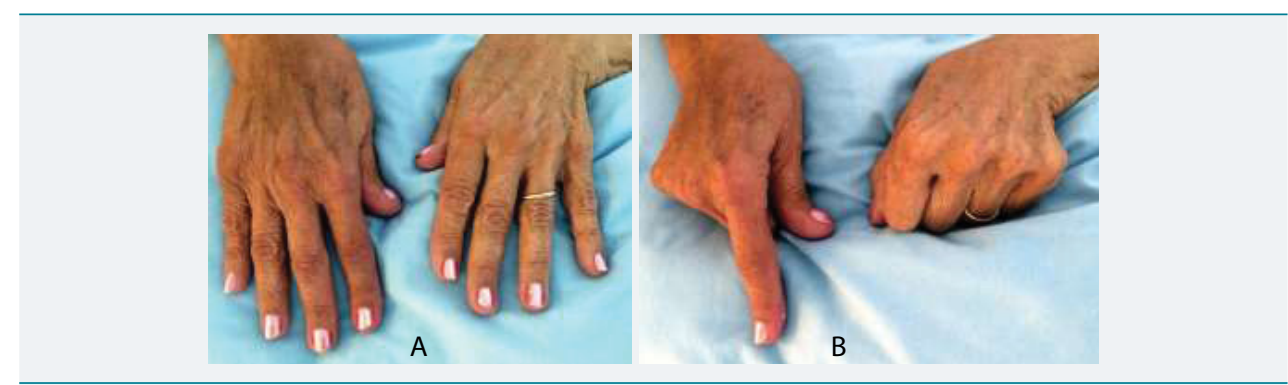

Figure 1: (A) Patient hands with fingers extended. (B) When asked to close her hands, the patient was unable to bend the first and second fingers of the right hand.

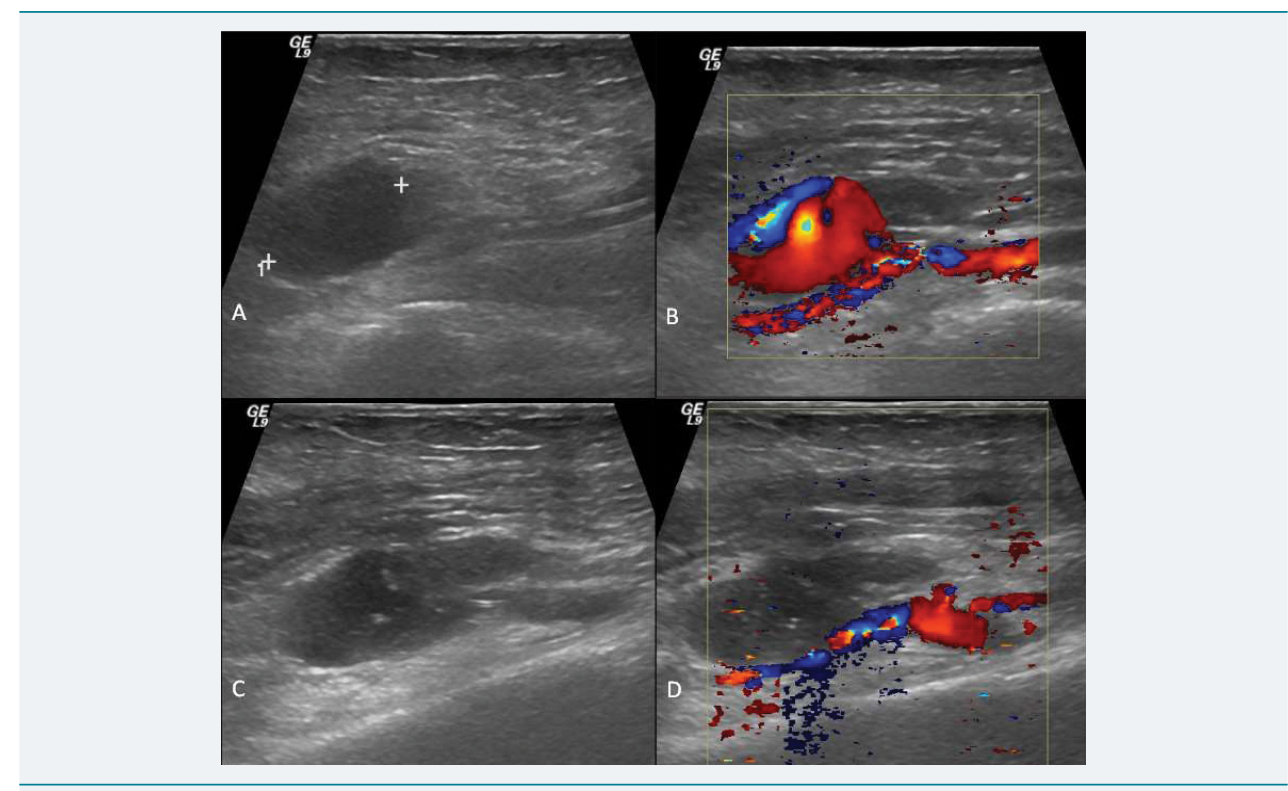

Figure 2: Sonographic images showing: (A) hypoechoic cystic image adjacent to the right brachial artery; (B) bidirectional flow pattern ("yin-yang sign"), typical of a pseudoaneurysm, identified on Doppler examination; (C) tip of the needle inside the pseudoaneurysm before thrombin injection (arrow); and (D) absence of flow on Doppler examination, confirming the pseudoaneurysm thrombosis.

two brachial veins, and, in close proximity to these vascular structures, the median nerve. Thus, injury to the artery and/or adjacent nerve is not unlikely when puncturing one of the veins. Eventualities such as the one described in this report have been rarely reported. However, these events can be extremely dangerous to the limb.

Accidental puncture of the brachial artery during PICC insertion should be identified and repaired immediately with appropriate local compression. Inadvertent insertion of the catheter into the arterial system with harmful consequences for the patient has been reported, because injection of hyperosmolar substances may result in thrombosis of the brachial artery, limb ischemia and stroke $[4,5]$, which can also be caused by catheter-related paradoxical embolism from venous thrombosis, as reported by Petrea et al. [6]. Thus, radiological control after PICC insertion in a deep vein should be used to determine and confirm correct tip placement in the superior vena cava [7].

Ultrasound-guided PICC line placement increases the success rate and reduces the incidence of vascular complications [8]. However, even under ultrasound control, catheter insertion resulted in a large pulsatile hematoma at the site of insertion, probably due to difficulty in advancing the dilator. The same difficulty was reported by Tran et al. (2006), which resulted in brachial arteriovenous fistula in the case described by the authors [2].

Even without obvious clinical signs of pseudoaneurysm in our patient, assessment $24 \mathrm{~h}$ post-insertion by a nurse in the PICC group, who referred the patient for a Doppler 
ultrasonography, was crucial for diagnosis of the arterial complication and eliminated the need for more complex imaging tests for diagnosis and therapy determination.

Spontaneous thrombosis of pseudoaneurysms $<3 \mathrm{~cm}$ in diameter were observed in $87 \%$ of cases reported by Toursarkissian et al. [9], an expectant approach did not appear appropriate in our case because of the associated nerve injury, which prompted immediate repair of the pseudoaneurysm to prevent possible compression of the nerve bundle. Because ultrasound-guided compression was not effective, we opted for thrombin injection into the pseudoaneurysm sac, which resulted in thrombosis of the pseudoaneurysm without negative repercussions to the main arterial axis.

The incidence of nerve lesions in percutaneous procedures is higher in the upper limbs than in the lower limbs because the brachial vascular structures are closely associated with the median nerve in the same fascial compartment. In a study that investigated the complications of cardiac catheterization via the brachial artery for coronary angiography, Kennedy et al. (1997) reported five cases of median nerve damage in 350 procedures performed, and the incidence of this complication was between 0.2 and $1.4 \%$. The authors identified three mechanisms of injury: direct nerve compression due to formation of antecubital fossa hematoma, direct nerve trauma by inappropriately placed needle tips, and ischemia secondary to brachial artery occlusion [10]. The outcome of this adverse event during PICC placement, although uncommon [3], affects the flexor muscles of the first and second fingers, and sometimes also of the third finger. Fascial compartment decompression is indicated when nerve compression due to formation of a hematoma is identified, but this course of action is unable to reverse the functional deficit of the hand, which is usually long-term but may improve with time. In our patient, the neurological deficit persisted four months after the procedure despite physical therapy. Two mechanisms of median nerve damage are suggested: compression by the pseudoaneurysm or direct trauma by the needle tip. Moreover, repair of the pseudoaneurysm by thrombin injection resulted in no functional improvement to the hand.

PICC line insertion is not without complications, which although uncommon, can be severe and disabling. This procedure should be ultrasound-guided, which not only increases success rates but also reduces the incidence of vascular complications. Moreover, because PICC insertion is painful and uncomfortable for the patient, the procedure should only be done with at least local anesthesia.

Finally, regulating and standardizing PICC line placement practices among specialized staff is critical to ensuring quality of care, according to the best scientific evidence and in compliance with current regulations.

\section{Acknowledgment}

This manuscript was reviewed by a professional science editor and by a native English-speaking copy editor to improve readability.

\section{References}

1. Chopra V, Anand S, Hickner A, Buist M, Rogers MA, Saint S, Flanders SA. Risk of venous thromboembolism associated with peripherally inserted central catheters: a systematic review and meta-analysis. Lancet. 2013; 382(9889): 311-325. Ref.: https://bit.ly/2QFdVvj

2. Tran HS, Burrows BJ, Zang WA, Han DC. Brachial arteriovenous fistula as a complication of placement of a peripherally inserted central venous catheter: a case report and review of the literature. Am Surg. 2006; 72: 833-836. Ref.: https://bit.ly/2Xmjy41

3. Alomari A, Falk A. Median nerve bisection: a morbid complication of a peripherally inserted central catheter. J Vasc Access. 2006; 7: 129-131. Ref.: https://bit.ly/2WazViG

4. Parikh S, Narayanan V. Misplaced peripherally inserted central catheter: an unusual cause of stroke. Pediatr Neurol. 2004; 30: 210-212 Ref.: https://bit.ly/2JS6joM 
5. Garg N, Noheria A, McPhail IR, Ricotta JJ 2nd. Embolic strokes after peripherally inserted central catheter placement. Ann Vasc Surg 2010; 24: 1133. e1- 1133.e4. Ref.: https://bit.ly/2HQL8B7

6. Petrea RE, Koyfman F, Pikula A, Romero JR, Viereck J, et al. Acute stroke, catheter related venous thrombosis, and paradoxical cerebral embolism: report of two cases. J Neuroimaging. 2013; 23: 111-114. Ref.: https://bit.ly/2KnHP6f

7. Amerasekera SS, Jones CM, Patel R, Cleasby MJ. Imaging of the complications of peripherally inserted central venous catheters. Clin Radiol. 2009; 64: 832-840 Ref.: https://bit.ly/2WJds0s

8. Bunting J, Slaughter RE, Masel PJ, Kroll WJ, and Bell SC. Ultrasound placement of peripherally inserted central catheters (PICCs) in adults with cystic fibrosis. Thorax. 2000; 55: 535. Ref.: https://bit.ly/2XI6mwk

9. Toursarkissian B, Allen BT, Petrinec D, Thompson RW, Rubin BG, et al. Spontaneous closure of selected iatrogenic pseudoaneurysms and arteriovenous fistulae. J Vasc Surg. 1997; 25: 803-808. Ref.: https://bit.ly/2EPc2Y6

10. Kennedy AM, Grocott $M$, Schwartz MS, Modarres $H$, Scott $M$, et al. Median nerve injury: an underrecognised complication of brachial artery cardiac catheterization. J Neurol Neurosurg Psychiatry. 1997; 63: 542-546. Ref.: https://bit.ly/2MnomFm 